\title{
Thermodynamics of fiber bundles
}

\author{
Steven R. Pride ${ }^{\mathrm{a}, *}$, Renaud Toussaint ${ }^{\mathrm{b}}$ \\ ${ }^{a}$ Géosciences Rennes, Université de Rennes 1, 35042 Rennes Cedex, France \\ ${ }^{\mathrm{b}}$ Dept. of Physics, University of Oslo, P.O.Box 1048 Blindern, 0316 Oslo, Norway
}

\begin{abstract}
A recent theory that determines the properties of disordered solids as the solid accumulates damage is applied to the special case of fiber bundles with global load sharing and is shown to be exact in this case. The theory postulates that the probability of observing a given emergent damage state is obtained by maximizing the emergent entropy as defined by Shannon subject to energetic constraints. This theory yields the known exact results for the fiber-bundle model with global load sharing and holds for any quenched-disorder distribution. It further defines how the entropy evolves as a function of stress, and shows definitively how the concepts of temperature and entropy emerge in a problem where all statistics derive from the initial quenched disorder. A previously unnoticed phase transition is shown to exist as the entropy goes through a maximum. In general, this entropy-maximum transition occurs at a different point in strain history than the stress-maximum transition with the precise location depending entirely on the quenched-disorder distribution.
\end{abstract}

Key words: Fiber Bundles, Entropy Maximization, Phase Transitions PACS: 46.50.+a, 46.65.+g, 62.20.Mk, 64.60.Fr

\section{INTRODUCTION}

The fiber-bundle model with global load sharing is a simple model for failure in tension introduced almost 80 years ago [1-3] and having received considerable attention and extensions over the past 15 years [4-19]. Although this model may have little pertinence to real fibrous systems such as a rope breaking in tension, it is of interest because it possesses exact analytical properties.

\footnotetext{
* Corresponding author. Fax: + 33223236090

Email addresses: Steve.Pride@univ-rennes1.fr (Steven R. Pride), Renaud.Toussaint@fys.uio.no (Renaud Toussaint).
} 
In recent work [20-22], we have developed a general statistical theory for determining the properties of a disordered solid that is accumulating irreversible damage due to cracking under stress. The ensembles in this theory are created by considering different realizations of the quenched disorder in the local breaking strength of the material. Such realizations are made either for the system as a whole, or of more pertinence to real systems, by dividing a given system into smaller "mesovolumes" and letting each mesovolume correspond to a different realization of the quenched disorder. The ensembles so obtained have nothing to do with thermal fluctuations (molecular dynamics). Given a system with such quenched disorder, our theory determines the probability of emergent crack states by maximizing Shannon's measure of disorder subject to constraints coming from the energetics of the fracture process.

In the present paper, we apply this theory to the specific problem of fiber bundles with global load sharing and demonstrate that for any quenched-disorder distribution, it yields the known exact results. Furthermore, a previously unnoticed phase transition is demonstrated to exist where entropy goes through a maximum. This phase transition is distinct from the well-known stress maximum transition and was noticed in the present theory because of the prominent role played by entropy.

However, the importance of our theory is not that it yields a new result in this old model, but that it applies and yields analytical results about phase transitions for any quasi-static damage model; albeit, for models involving crack interactions, approximate treatment of the functional integrations may be required to obtain analytical results (such as renormalization or mean-field approximations). Using our theory, we recently treated the problem of how the mechanical properties of rocks change due to cracks arriving and interacting in compressive shear [20-22]. We analytically demonstrated that the localization transition observed in experiments is a second-order phase transition when it occurs.

\section{THERMODYNAMICS}

Our theory was originally developed for a disordered solid under the influence of a stress tensor. For fiber bundles, a much simpler scalar theory applies and so in this section, the formalism is rederived in this simplified context.

A fiber bundle is depicted in Fig. 1. A collection of $N$ fibers are stretched between two rigid supports. One support is held fixed, while the other is free to displace. A load $F_{N}$ is applied to the bundle through the free support so that the fibers are in a state of tension. In this paper, the load $F_{N}$ will always be normalized by $N$ to define the overall tension $\tau=F_{N} / N$. Each non-broken 


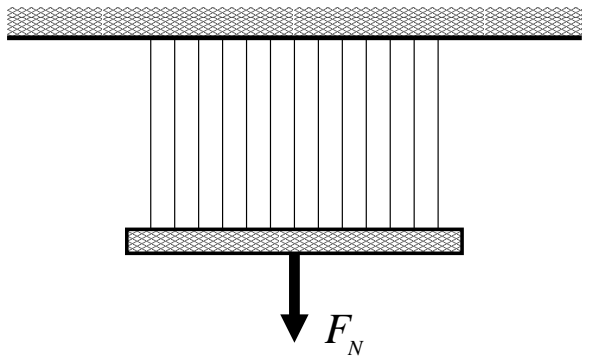

Fig. 1. A bundle of $N$ fibers stretched between two rigid supports with a load $F_{N}$ applied through the free support.

fiber in a bundle has the same length $L$. If, when $\tau=0$, this length is $L_{o}$, then the measure of strain is $\varepsilon=L / L_{o}-1$. Experiments may be performed on the bundle either by controlling $\tau$ or $\varepsilon$.

Each of the fibers has the same Young's modulus which is taken to be unity so that the axial strain $\varepsilon$ of each fiber is identical to the tension in the fiber. The $N$ fibers have strengths $\varepsilon_{1}, \varepsilon_{2}, \ldots \varepsilon_{N}$ which are independent random variables sampled from a distribution $p(\varepsilon)$, whose cumulative distribution is defined $P(\varepsilon)=\int_{0}^{\varepsilon} p(x) d x$. As the strain $\varepsilon$ of the bundle is increased, the individual fibers will break once their tension (strain) gets to their fixed strength threshold. The assumption of "global load sharing" is that when one of the fibers breaks at fixed load, all the other fibers will extend by the same amount thus increasing the tension in each of the surviving fibers so that the load as a whole is always supported entirely by the surviving fibers. All of this defines the fiber bundle model with global load sharing. Of interest are the mechanical properties of such bundles as averaged over all possible realizations of the fiber strengths.

We first need to know the probability $p_{j}$ of observing one of the realizations to be in a particular state $j$ of damage when the ensemble as a whole is at an applied strain $\varepsilon$. In the fiber bundle model, a damage state $j$ is defined by which of the $N$ fibers are broken. One could define $j$ using a local order parameter that is 1 if a fiber is intact and 0 if the fiber is broken.

Our theory postulates that the fraction $p_{j}$ of all realizations observed to be in state $j$ is obtained by maximizing Shannon's measure of disorder

$$
S=-\sum_{j} p_{j} \ln p_{j}
$$

subject to constraints. Such constraints must involve the independent variables of $S$. To identify the independent variables, we consider how both $S$ and the average energy in the ensemble of bundles change as the strain is increased.

When $\varepsilon$ increases to $\varepsilon+d \varepsilon$, there is both a work carried out in reversibly stretching the fibers and an additional work carried out due to irreversible 
fiber breaks. Due to breaking, some of the members of the ensemble (individual realizations of the disorder) will be led out of their current damage state and into state $j$, while others that were in state $j$ will transfer to still different states. If there is a difference in the number of members entering and leaving state $j$, there will be a change $d p_{j}$ in the occupation probability of state $j$ and such changes are what cause Shannon's disorder measure $S$ to change.

The average energy density (average energy normalized by $N$ ) in the ensemble is given by $U=\sum_{j} p_{j} E_{j}$. Here, $E_{j}$ is the energy density required to create state $j$ at imposed strain $\varepsilon$ and as averaged over all members that have been led to state $j$. Depending on the breaking strengths of a given realization, the work performed in arriving at state $j$ can be different. It is through $E_{j}$ that all dependence on the quenched-disorder distribution enters the problem. The change that occurs when $\varepsilon$ increases to $\varepsilon+d \varepsilon$ is

$$
d U=\sum_{j} E_{j} d p_{j}+\sum_{j} p_{j} d E_{j}
$$

The first term is the energy expended in changing the disorder over the collection of realizations. It is thus proportional to the disorder change and can be written

$$
T d S=\sum_{j} E_{j} d p_{j}
$$

The second term is written

$$
f d \varepsilon=\sum_{j} p_{j} d E_{j}
$$

and represents both the reversible stretching energy in those members that did not experience breaks during the deformation increment, as well as the irreversible energy changes due to all the breaks that did not result in a net change in the occupation numbers of each state.

This decomposition of the energy increment can be thought of as follows. When breaks occur throughout the ensemble of realizations as $\varepsilon$ increases to $\varepsilon+d \varepsilon$, there is a flow of members between the states. This flow involves energy changes due to fibers breaking in the interval $d \varepsilon$. It may be resolved into a uniform "incompressible" part whose associated energy is included within $f d \varepsilon$ as well as a non-uniform "compressible" part associated with more members arriving in a given state than are leaving that state. The energy associated with the non-uniform flow between states is entirely contained in $T d S$.

From these expressions it may be concluded that if $U$ is to be treated as a fundamental function, then $U=U(S, \varepsilon)$, or equivalently if $S$ is to be treated as the fundamental function then $S=S(U, \varepsilon)$. In other words, the independent variables that must be involved in the constraints on the maximization of $S$ 
are $U$ and $\varepsilon$. The proportionality constants $T$ and $f$ are defined

$$
T=\left(\frac{\partial U}{\partial S}\right)_{\varepsilon} \text { and } f=\left(\frac{\partial U}{\partial \varepsilon}\right)_{S} .
$$

The state function $f$ is something different than the overall tension $\tau$ since we also have that $\tau d \varepsilon=d U$. Thus, in general, $(\tau-f) d \varepsilon=T d S$ so that $f \neq \tau$ due to fibers breaking in a positive increment $d \varepsilon$. If strain were to be decreased, fibers do not break and so $d S=0$ and the state function $f$ would be defined using only the purely elastic part of the energy changes $d E_{j}$. Changes in $f$ in this case are equivalent to changes in $\tau$. Last, since we have taken $S$ to be extensive (proportional to $N$ ) while $U$ is a density independent of $N, T$ comes out being proportional to $1 / N$. This choice is made so that factors of $N$ do not clutter the equations that follow.

The constraint involving $\varepsilon$ is that each non-broken fiber throughout the entire ensemble has the same length which implies $\varepsilon_{j}=\varepsilon$. The constraint involving $U$ is that $U=\sum_{j} p_{j} E_{j}$. Carrying out the maximization of $S$ subject to these constraints using Lagrangian multipliers gives the probability distribution as

$$
p_{j}(\beta, \varepsilon)=\frac{e^{-\beta E_{j}(\varepsilon)}}{Z(\beta, \varepsilon)}
$$

where $\beta=1 / T$ and where the partition function $Z$ is defined

$$
Z(\beta, \varepsilon)=\sum_{j} e^{-\beta E_{j}(\varepsilon)}
$$

The free energy $F=F(\beta, \varepsilon)$ in this ensemble (for this set of constraints) is the Legendre transform of $U$ with respect to $S$; i.e., $F=U-T S$. Differentiation then gives

$$
d F=\beta^{-2} S d \beta+f d \varepsilon .
$$

Upon introducing $U=\sum_{j} p_{j} E_{j}$ and $S=-\sum_{j} p_{j} \ln p_{j}$ into the Legendre transform $F=U-S / \beta$ and using that $\ln p_{j}=-\beta E_{j}-\ln Z$ we obtain the standard result

$$
\beta F=-\ln Z \text {. }
$$

Thus, the standard canonical ensemble emerges in this problem where quenched disorder alone (not molecular fluctuations) is responsible for the existence of ensembles.

An important question in such an approach is whether anything precise can be said about the temperature $T=1 / \beta$. Indeed, $\beta$ can formally be found as the solution of a differential equation. This differential equation is obtained from the previously stated but unused fact that $d U=\tau d \varepsilon$ which can be written

$$
\tau=\frac{d U}{d \varepsilon}=\sum_{j} \frac{d p_{j}}{d \varepsilon} E_{j}+\sum_{j} p_{j} \frac{d E_{j}}{d \varepsilon} .
$$




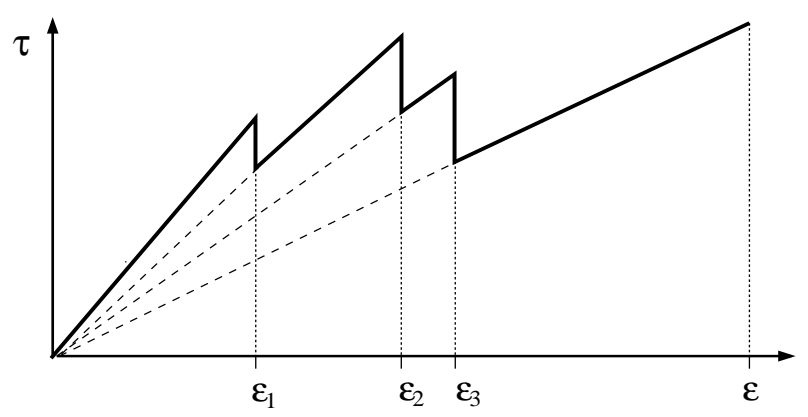

Fig. 2. A strain-controlled experiment. The solid line is the load path followed during the experiment. The dashed lines represent the path that would be followed if strain were to be decreased at some point during the experiment. When a fiber breaks at constant $\varepsilon$, the load $\tau$ must be reduced as represented by the vertical drops.

Now from Eq. (6), we obtain that

$$
\frac{d p_{j}}{d \varepsilon}=\left[-\frac{d E_{j}}{d \varepsilon} \beta-E_{j} \frac{d \beta}{d \varepsilon}-\frac{d \ln Z}{d \varepsilon}\right] p_{j},
$$

while from Eqs. (8) and (9)

$$
\frac{d \ln Z}{d \varepsilon}=-F \frac{d \beta}{d \varepsilon}-\beta \frac{d F}{d \varepsilon}=-U \frac{d \beta}{d \varepsilon}-\beta f .
$$

Since each member is at the same $\varepsilon$, each member has its own $\tau_{j}$, and so $\tau=\sum_{j} p_{j} \tau_{j}$. We then obtain the differential equation for $\beta$ in the form

$$
a \frac{d \beta}{d \varepsilon}+b \beta+c=0
$$

with coefficients given by

$$
a=\sum_{j} p_{j} E_{j}\left(U-E_{j}\right) ; \quad b=\sum_{j} p_{j} E_{j}\left(f-\frac{d E_{j}}{d \varepsilon}\right) ; c=\sum_{j} p_{j}\left(\frac{d E_{j}}{d \varepsilon}-\tau_{j}\right) .
$$

Since $p_{j}=p_{j}(\beta, \varepsilon)$, this equation is non-linear and thus difficult to solve. In the present work, we demonstrate that a proposed function $\beta=\beta(\varepsilon)$ exactly satisfies this equation and thus is the true "fiber-bundle" $\beta$. To make progress, we next obtain $E_{j}$ and $\tau_{j}$ for the specific problem of fiber bundles with globalload sharing.

\section{FIBER BUNDLE MODEL}

Figure 2 details the history of how the overall tension $\tau$ might evolve when controlled variations in $\varepsilon$ are applied to a bundle having fiber strengths $\varepsilon_{1}, \varepsilon_{2} \ldots \varepsilon_{N}$. In this particular example, $n=3$ fibers have broken when the strain is at $\varepsilon$. 
The load on the bundle is equally shared by the $N-n$ surviving fibers so that

$$
\tau_{n}=\varepsilon\left(1-\frac{n}{N}\right)
$$

which is a relation independent of the history; i.e. it depends only on the actual state of the bundle through the number of broken fibers $n$, and not on the breaking thresholds $\varepsilon_{1}, \varepsilon_{2} \ldots \varepsilon_{n}$.

The total work density $E_{j}^{p}$ for states $j$ consisting of $n$ broken fibers is the area under the charging curve in a particular realization (the area under the solid line in Fig. 2)

$$
E_{j}^{p}=\int_{0}^{\varepsilon} \tau(x) d x=\sum_{m=0}^{n} \int_{\varepsilon_{m}}^{\varepsilon_{m+1}} \tau_{m}(x) d x=\sum_{m=0}^{n}\left(1-\frac{m}{N}\right)\left(\frac{\varepsilon_{m+1}^{2}}{2}-\frac{\varepsilon_{m}^{2}}{2}\right)
$$

where from Eq. (15) we have used $\tau_{m}(x)=x(1-m / N)$ and where by convention $\varepsilon_{n+1}=\varepsilon$ is the final applied strain. A direct recursion gives exactly

$$
E_{j}^{p}=\left(1-\frac{n}{N}\right) \frac{\varepsilon^{2}}{2}+\sum_{m=1}^{n} \frac{\varepsilon_{m}^{2}}{2 N} .
$$

The first term here is the elastic energy that can be reversibly recovered upon decreasing the strain while the second term represents the energy irreversibly consumed in the breaking process. Both contributions can be directly vizualized in Fig. 2.

Equation (16) is next averaged over the quenched disorder to obtain the average energy $E_{j}$ needed to create state $j$. Each breaking threshold $\varepsilon_{m}$ is an independant variable, randomly distributed according to $p\left(\varepsilon_{m}\right)$ under the condition that $0 \leq \varepsilon_{m} \leq \varepsilon$. They are therefore distributed according to the probability density $p\left(\varepsilon_{m}\right) / P(\varepsilon)$ where the normalization factor accounts for the fact that the upper limit $\varepsilon$ is independent of the threshold values $\varepsilon_{m}$. Thus, averaging over the quenched disorder gives

$$
h(\varepsilon)=\left\langle\frac{\varepsilon_{m}^{2}}{2}\right\rangle_{\text {q.d. }}=\frac{1}{P(\varepsilon)} \int_{0}^{\varepsilon} \frac{x^{2}}{2} p(x) d x
$$

where $h(\varepsilon)$ designates the average energy that is lost when each fiber breaks.

The average work density (Hamiltonian) required to create the state $j$, averaged over all realizations of the quenched disorder is then in general

$$
E_{j}=\left(1-\frac{n_{j}}{N}\right) \frac{\varepsilon^{2}}{2}+\frac{n_{j}}{N} h(\varepsilon)=\frac{\varepsilon^{2}}{2}-\frac{n_{j}}{N}\left[\frac{\varepsilon^{2}}{2}-h(\varepsilon)\right] .
$$

For example, under the special assumption that the breaking strengths are randomly sampled from a uniform distribution on $0 \leq \varepsilon_{m} \leq 1$, we have that $p(\varepsilon)=1, P(\varepsilon)=\varepsilon, h(\varepsilon)=\varepsilon^{2} / 6$, and $E_{j}=\varepsilon^{2} / 2-\left(n_{j} / N\right) \varepsilon^{2} / 3$. 


\section{Average Properties}

We now apply the above theory and determine the thermodynamic functions as a function of applied strain $\varepsilon$. The following analysis is valid for any properly normalized quenched-disorder distribution $p(\varepsilon)$.

The cumulative distribution $P(\varepsilon)=\int_{0}^{\varepsilon} p(x) d x$ is the probability that any one fiber has broken when the strain is at $\varepsilon$. Thus, the fraction of all possible realizations having $n_{j}$ broken fibers and $N-n_{j}$ unbroken fibers is exactly

$$
p_{j}^{\text {exact }}=(1-P)^{N-n_{j}} P^{n_{j}}
$$

This distribution may be written

$$
p_{j}^{\text {exact }}=p_{0} \exp \left[-n_{j} \ln \left(\frac{1-P}{P}\right)\right]
$$

where $p_{0}=(1-P)^{N}$ is the probability of the unbroken state $j=0$. Upon using the Hamiltonian of Eq. (18), the postulate of entropy maximization predicts this same distribution to be given by

$$
p_{j}=p_{0} \exp \left[n_{j} \frac{\beta}{N}\left(\frac{\varepsilon^{2}}{2}-h\right)\right]
$$

where $p_{0}=\exp \left(-\beta \varepsilon^{2} / 2\right) / Z$ is the probability of the unbroken state.

These two distributions are both Boltzmannians in the number $n_{j}$ of broken fibers and are identical if the temperature $T=1 / \beta$ is given by

$$
\beta(\varepsilon)=\frac{-N}{\varepsilon^{2} / 2-h(\varepsilon)} \ln \left(\frac{1-P(\varepsilon)}{P(\varepsilon)}\right) .
$$

If it can be shown that this $\beta$ satisfies the differential equation of Eq. (13), then our theory is exact when applied to fiber bundles with global load sharing.

From the definition $P(\varepsilon) h(\varepsilon)=\int_{0}^{\varepsilon} p(x) x^{2} / 2 d x$, one has $h(\varepsilon)<\varepsilon^{2} / 2$ for any distribution $p(\varepsilon)$. Thus, $\beta(\varepsilon)$ is a negative increasing function up to the strain point $\varepsilon=\varepsilon_{\beta}$ where it smoothly goes to zero. The inflection point $\varepsilon_{\beta}$ is obtained from the condition that $P\left(\varepsilon_{\beta}\right)=1 / 2$ and defines a previously unnoticed phase transition that will be shown to be distinct from the transition at peak stress. When $\varepsilon>\varepsilon_{\beta}, \beta$ becomes a positive increasing function of $\varepsilon$.

There are two key average properties upon which all the thermodynamic functions depend; namely, the average fraction of broken fibers in each bundle $\left\langle n_{j} / N\right\rangle$ and the average of this fraction squared $\left\langle\left(n_{j} / N\right)^{2}\right\rangle$. Using the exact probabilities of Eq. (20) [which is equivalent to using the $\beta$ of Eq. (22) in our 
probability law], one obtains

$$
\left\langle\frac{n_{j}}{N}\right\rangle=\sum_{j} p_{j} \frac{n_{j}}{N}=\sum_{n=0}^{N} c_{n}^{N} \frac{n}{N} P^{n}(1-P)^{N-n}
$$

where $c_{n}^{N}=N ! /[n !(N-n) !]$ defines the number of ways of selecting $n$ objects from a group of $N$ distinguishable items. The binomial theorem states that

$$
(x+y)^{N}=\sum_{n=0}^{N} c_{n}^{N} x^{n} y^{N-n} .
$$

Upon differentiating this equation with respect to $x$ and then multiplying by $x / N$, gives that when $x=P$ and $y=1-P$

$$
\left\langle\frac{n_{j}}{N}\right\rangle=P
$$

which is a known result consistent with the meaning of $P$. Differentiating a second time yields

$$
\left\langle\left(\frac{n_{j}}{N}\right)^{2}\right\rangle=P^{2}+\frac{P(1-P)}{N} .
$$

Using these two results, the other averages defining the thermodynamic variables are easily read off.

The average stress $\tau(\varepsilon)$ is thus obtained to be

$$
\tau=\sum_{j} p_{j} \tau_{j}=(1-P) \varepsilon
$$

which is initially an increasing function of $\varepsilon$ having the slope

$$
\frac{d \tau}{d \varepsilon}=1-P-\varepsilon p
$$

This slope goes to zero and the stress has a maximum at any strain point $\varepsilon_{\tau}$ that is a solution of $1-P\left(\varepsilon_{\tau}\right)-\varepsilon_{\tau} p\left(\varepsilon_{\tau}\right)=0$; i.e., at the point(s) where

$$
\varepsilon_{\tau} p\left(\varepsilon_{\tau}\right)=1-\int_{0}^{\varepsilon_{\tau}} p(x) d x
$$

admits a solution. This is a known exact result $[4,10]$. In general, we can expect that $\varepsilon_{\tau} \neq \varepsilon_{\beta}$. The condition required for equality of these strain points is that simultaneously $\varepsilon p(\varepsilon)=1 / 2$ and $\int_{0}^{\varepsilon} p(x) d x=1 / 2$ which for monotonic distribution functions $p(\varepsilon)$ can only occur with the uniform distribution $p(\varepsilon)=$ 1 in which case $\varepsilon_{\tau}=\varepsilon_{\beta}=1 / 2$ and the two transitions coincide. If $p(\varepsilon)$ is a monotonic increasing function of $\varepsilon$, then $\varepsilon_{\tau}<\varepsilon_{\beta}$ while if it is a decreasing function of $\varepsilon$, then $\varepsilon_{\tau}>\varepsilon_{\beta}$. For non-monotonic distributions, there can be an arbitrary number of stress maximas and Eq. (29) can have either no solutions 
or multiple solutions [10]. The nature of the phase transitions at the distinct strain points $\varepsilon_{\beta}$ and $\varepsilon_{\tau}$ is discussed in the following section.

The average energy in the ensemble is

$$
U=\sum_{j} p_{j} E_{j}=(1-P) \frac{\varepsilon^{2}}{2}+h P .
$$

Again recalling the definition of $h$ from Eq. (17) gives

$$
\frac{d U}{d \varepsilon}=(1-P) \varepsilon-p \frac{\varepsilon^{2}}{2}+\frac{d(h P)}{d \varepsilon}=\tau
$$

which is also the equation that gives the differential equation for temperature. This is sufficient for demonstrating that the $\beta(\varepsilon)$ of Eq. (22) satisfies its differential equation. Nonetheless, as a consistency test, the coefficients $a, b$, and $c$ defined in Eq. (14) will be derived and the differential equation will explicitly be shown to be satisfied.

To obtain the state function $f$, we need first

$$
\frac{d E_{j}}{d \varepsilon}=\varepsilon-\frac{n_{j}}{N}\left[\varepsilon-\frac{p}{P}\left(\frac{\varepsilon^{2}}{2}-h\right)\right] .
$$

From Eq. (4) and the lemma of Eq. (25) we then have

$$
f=\sum_{j} p_{j} \frac{d E_{j}}{d \varepsilon}=(1-P) \varepsilon+p\left(\frac{\varepsilon^{2}}{2}-h\right) .
$$

The variation of the entropy is obtained from the energy balance as

$$
\frac{d S}{d \varepsilon}=\beta\left(\frac{d U}{d \varepsilon}-f\right)=N p \ln \left(\frac{1-P}{P}\right) .
$$

Together with the initial condition $S(0)=0$, this is readily integrated to give

$$
S=-N[P \ln P+(1-P) \ln (1-P)] .
$$

This expression is the classical Shannon result for a set of $N$ random variables in two possible states having probabilities $P$ and $1-P$, which is precisely the case of the fiber bundle with global sharing. This is another consistency check. This entropy $S$ increases from zero [total certainty that every member is intact] and goes smoothly through a maximum [total uncertainty as to what state a member may be in] at the same strain point $P\left(\varepsilon_{\beta}\right)=1 / 2$ where $\beta$ goes to zero. After the smooth maximum, $S$ decreases to reach zero if $P(\varepsilon)$ reaches 1 in which case there is total certainty that each member is entirely broken. We note that Eq. (35) can also be directly obtained from the Shannon formula upon applying the binomial theorem. 
Finally, the free energy $F$ is again obtained from its Legendre transform definition $F=U-S / \beta$ to be

$$
F=\frac{\varepsilon^{2}}{2}-\left(\frac{\varepsilon^{2}}{2}-h\right) \frac{\ln (1-P)}{\ln (1-P)-\ln P} .
$$

At the point $\varepsilon_{\beta}$ defined by $P\left(\varepsilon_{\beta}\right)=1 / 2$, the free energy diverges due to the fact that $\beta\left(\varepsilon_{\beta}\right)=0$ while $S\left(\varepsilon_{\beta}\right)$ remains finite. So long as $\varepsilon_{\beta} \neq \varepsilon_{\tau}$, the free energy does not diverge when (if) $\varepsilon=\varepsilon_{\tau}$. In passing, we also note that

$$
Z=\left[(1-P)^{h} P^{-\varepsilon^{2} / 2}\right]^{N /\left(\varepsilon^{2} / 2-h\right)}
$$

is the exact expression of the partition function.

With the above results established, we now obtain the coefficients $a, b, c$ of the differential equation for the temperature as

$$
\begin{aligned}
& a=-\frac{1-P}{N}\left(P h^{2}-\frac{\varepsilon^{2}}{2}\right)^{2} ; b=\frac{1-P}{N}\left(P h^{2}-\frac{\varepsilon^{2}}{2}\right)\left(P \varepsilon-p \frac{\varepsilon^{2}}{2}+p h\right) ; \\
& c=-p\left(h-\frac{\varepsilon^{2}}{2}\right) .
\end{aligned}
$$

Using these together with Eq. (22) for $\beta$ and its derivative

$$
\frac{d \beta}{d \varepsilon}=-\frac{N p}{P(1-P)\left(h-\varepsilon^{2} / 2\right)}+N \ln \left(\frac{1-P}{P}\right)\left[\frac{\varepsilon+\left(h-\varepsilon^{2} / 2\right) p / P}{\left(h-\varepsilon^{2} / 2\right)^{2}}\right]
$$

shows that the differential equation $a d \beta / d \varepsilon+b \beta+c=0$ is exactly satisfied.

\section{Phase Transitions}

\subsection{The entropy maximum transition}

The strain point $\varepsilon_{\beta}$ defined by $P\left(\varepsilon_{\beta}\right)=1 / 2$ is where simultaneously $\beta=0$, the entropy is a maximum, and the free energy diverges. It is distinct from the stress-maximum transition(s) at $\varepsilon_{\tau}$. The interpretation of $\varepsilon=\varepsilon_{\beta}$ as a phase transition is natural, since the most probable configuration of a bundle suddenly jumps from being entirely intact to entirely broken. What are the measurable manifestations of this transition at $\varepsilon_{\beta}$ ?

Since the entropy is a maximum at this point, the fluctuations between realizations should also be a maximum as we now demonstrate. Define the fraction of 
broken fibers in state $j$ to be $\rho_{j}=n_{j} / N$, and define the average of this fraction to be $\rho=\left\langle\rho_{j}\right\rangle=P$ where $P=P(\varepsilon)$ is again the probability of a fiber being broken. The quantity of interest here is the root-mean-square fluctuation $\Delta \rho$ in the fraction of broken fibers given by

$$
\Delta \rho=\sqrt{\left\langle\Delta \rho_{j}^{2}\right\rangle}=\sqrt{\left\langle\rho_{j}^{2}\right\rangle-\rho^{2}}=\frac{\sqrt{P(1-P)}}{\sqrt{N}}
$$

which indeed goes through a maximum at $P\left(\varepsilon_{\beta}\right)=1 / 2$ as expected. This maximum is something that can be directly measured in numerical experiments on fiber bundles but has never before been commented on. The reason it has been discovered in the present theory is because entropy and temperature are explicitly present. We encourage someone to numerically measure $\Delta \rho$ and to verify that it is a maximum at the transition point $\varepsilon_{\beta}$. Recall that for monotonic quenched-disorder distributions, if $p(\varepsilon)$ is a decreasing function (more weak fibers than strong fibers), then $\varepsilon_{\beta}<\varepsilon_{\tau}$. So the numerical observation in this case should be that $\Delta \rho$ goes through a maximum prior to peak stress. Equivalent comments hold when $p(\varepsilon)$ is an increasing function and $\varepsilon_{\beta}>\varepsilon_{\tau}$.

The other fluctuations that are potentially of interest include the root-meansquare stress fluctuation

$$
\Delta \tau=\sqrt{\left\langle\Delta \tau_{j}^{2}\right\rangle}=\sqrt{\left\langle\tau_{j}^{2}\right\rangle-\tau^{2}}=\varepsilon \Delta \rho
$$

and the root-mean-square energy fluctuation

$$
\Delta U=\sqrt{\left\langle\Delta E_{j}^{2}\right\rangle}=\sqrt{\left\langle E_{j}^{2}\right\rangle-U^{2}}=\left(\frac{\varepsilon^{2}}{2}-h\right) \Delta \rho,
$$

but since these are simply proportional to $\Delta \rho$ it seems that the interesting signature of this phase transition is the maximum in $\Delta \rho$.

The "order" of this transition is not classically defined in the Ehrenfest scheme; however, it seems inappropriate to call it a continuous transition because the free energy is singular at $\varepsilon_{\beta}$ as shown above. Nonetheless, in the limit as $\varepsilon \rightarrow \varepsilon_{\beta}$ the singular part of $F$ diverges according to the scaling law

$$
F_{s}=-\frac{\ln 2}{8} \frac{\left[\varepsilon_{\beta}^{2}-h\left(\varepsilon_{\beta}\right) / 2\right]}{p\left(\varepsilon_{\beta}\right)}\left(\varepsilon-\varepsilon_{\beta}\right)^{-1}
$$

which has a (trivial) universal exponent. Since $F$ and its derivatives are difficult to numerically measure, the principle manifestation of this phase transition remains $\Delta \rho$ going through a maximum at a point $\varepsilon_{\beta} \neq \varepsilon_{\tau}$. 


\subsection{The stress maximum transition}

The phase transition at peak stress $\varepsilon=\varepsilon_{\tau}$ is the one that researchers up to now have focused on. Since $S$ and its derivatives remain continuous and finite there, an attempt to classify this transition as being first-order or second-order is meaningless. The label of "continuous" transition seems the most appropriate.

There is universality at this transition due to the fact that for any monotonic analytic quenched-disorder distribution, $\tau(\varepsilon)$ is a smooth analytic function so that upon developing this function in the neighborhood of its maximum one is guaranteed $\left|\tau-\tau\left(\varepsilon_{\tau}\right)\right| \sim\left|\varepsilon-\varepsilon_{\tau}\right|^{2}$ with the exponent of course being independent of the distribution $p(\varepsilon)$ or other model parameters. Such development of an analytic function about a critical point is the way any mean-field theory acquires a universal scaling law. However, Kloster, Hansen and Hemmer [10] do demonstrate that a non-analytic quenched-disorder distribution can lead to highly non-analytic stress-strain behavior that thus falls outside the quadratic universality class. Also, in other damage models in which elastic interaction between cracks (damage points) is important, the simple observation of a model exhibiting an averaged stress maximum does not by itself guarantee an exactly quadratic stress-strain relation in the neighborhood of the maximum. Such a relation may be non-analytic due to a diverging correlation length in the correlation between cracks.

The quadratic stress maximum means that $d \varepsilon / d \tau$ diverges as $\left|\tau-\tau\left(\varepsilon_{\tau}\right)\right|^{-1 / 2}$ $[11,19]$. Accordingly, the average rate at which the fraction of broken fibers increases with stress $d \rho / d \tau=p d \varepsilon / d \tau$ also diverges as $\left|\tau-\tau\left(\varepsilon_{\tau}\right)\right|^{-1 / 2}[4,15]$. Using additional considerations not developed in this paper, one can futher show that the average size of avalanches also diverges as $\left|\tau-\tau\left(\varepsilon_{\tau}\right)\right|^{-1 / 2}[10,15,17]$, and that the distribution $D(\Delta)$ of the size $\Delta$ of the avalanches scales as $D(\Delta) \sim \Delta^{-5 / 2}[6,7,10,17]$ at the stress maximum. These are the principal observable characteristics of the stress-maximum transition.

\section{Summary and Conclusions}

Two principal results have been obtained in this paper. First, it was demonstrated that for any quenched-disorder distribution used in the fiber-bundle model with global load sharing, the probability of the emergent damage states may be exactly calculated by maximizing Shannon's entropy subject to constraints. It is through the constraints that the nature of the quenched-disorder distribution enters the macroscopic thermodynamics.

Second, a previously unnoticed phase transition occurs in the fiber-bundle 
model with global load sharing when the entropy goes through a maximum. This phase transition is distinct from the stress-maximum transition; although, for the uniform quenched-disorder distribution, the two transitions coincide. The principle manifestation of this transition is that the root-mean-square fluctuation in the number of broken fibers will go through a maximum, which is a quantity that can be directly measured in numerical experiments.

To conclude, we postulate that the probability of emergent states can always be calculated through entropy maximization for any damage model of interest including models in which there is elastic interaction between the local damage (order) parameters. Damage models with order-parameter interactions are not normally considered amenable to analytical treament; however, using our approach a rather standard canonical-ensemble partition function emerges and the various functional integration procedures available for studying the partition function in the neighborhood of critical points may be employed.

Such generality is the principal utility of our approach. A concern is the ability to produce an expression for the model temperature. The temperature can be found in principle as the solution of a well-posed initial-value problem. Unfortunately, the differential problem is highly non-linear and thus difficult to solve. Because stress maxima and entropy maxima do not normally coincide, a stress-maximum transition may be studied by simply assuming the temperature to be well behaved in the neighborhood of the stress maxima. But if an explicit expression for the temperature is desired, the following approach can be employed.

In models involving crack interactions, there are always a subset of dilute states in which the interactions are negligible. The exact probabilities of such states can usually be determined from the quenched-disorder distribution alone. A comparison between the probabilities calculated from entropy maximization and such exact probabilities then determines the model temperature to be used in the Boltzmannian. With the temperature so defined, and with a proper model Hamiltonian in hand, the partition function can be analyzed.

\section{References}

[1] F.T. Peirce, J. Text. Ind. 17, 355 (1926).

[2] H.E. Daniels, Proc. R. Soc. A 183, 404 (1945).

[3] B.D. Coleman, J. Appl. Phys. 29, 968 (1958).

[4] J.V. Andersen, D. Sornette and K.W. Leung, Phys. Rev. Lett. 78, 2140 (1997).

[5] H.E. Daniels, Adv. Appl. Prob. 21, 315 (1989).

[6] A. Hansen and P.C. Hemmer, Phys. Lett. A 184, 394 (1994).

[7] P.C. Hemmer and A. Hansen, J. Appl. Mech. 59, 909 (1992). 
[8] R.C. Hidalgo, F. Kun, and H.J. Herrmann, Phys. Rev. E 65, 032502 (2002).

[9] R.C. Hidalgo, F. Kun, and H.J. Herrmann, Phys. Rev. E 64, 066122 (2001).

[10] M. Kloster, A. Hansen, and P.C. Hemmer, Phys. Rev. E 56, 2615 (1997).

[11] F. Kun, S. Zapperi, and H.J. Herrmann, Eur. Phys. J. B 17, 269 (2000).

[12] D. Krajcinovic, and M.A.G. Silva, Int. J. Solids Structures 18, 551 (1982).

[13] I.L. Menezes-Sobrinho, A.T. Bernardes, and J.G. Moreira, Phys. Rev. E 63, 025104(R) (2001).

[14] L. Moral, Y. Moreno and A.F. Pacheco, Phys. Rev. E 63, 066106 (2001).

[15] Y. Moreno, J.B. Gomez and A.F. Pacheco, Phys. Rev. Lett. 85, 2865 (2000).

[16] Y. Moreno, J.B. Gomez and A.F. Pacheco, Physica A 274, 400 (1999).

[17] S. Roux, A. Delaplace and G. Pijaudier-Cabot, Physica A 270, 35 (1999).

[18] S. Roux, Phys. Rev. E 62, 6164 (2000).

[19] D. Sornette, J. Phys. A 22, L243 (1989).

[20] R. Toussaint and S.R. Pride, Phys. Rev. E (2002), (in press).

[21] R. Toussaint and S.R. Pride, Phys. Rev. E (2002), (in press).

[22] R. Toussaint and S.R. Pride, Phys. Rev. E (2002), (in press). 